Commonplace • Series 1.3: Global Transition to Open

\title{
Aligning Open Knowledge Practices and Spending with Issues of DEI and Justice
}

\author{
Alexia Hudson-Ward
}

Published on: Nov 16, 2021

DOI: $10.21428 / 6 \mathrm{ffd} 8432.8 \mathrm{ee} 163 \mathrm{~b} 7$

License: Creative Commons Attribution 4.0 International License (CC-BY 4.0). 
The video is captioned and there's a transcript of the presentation below. Please make comments on the transcript for further conversation and idea sharing!

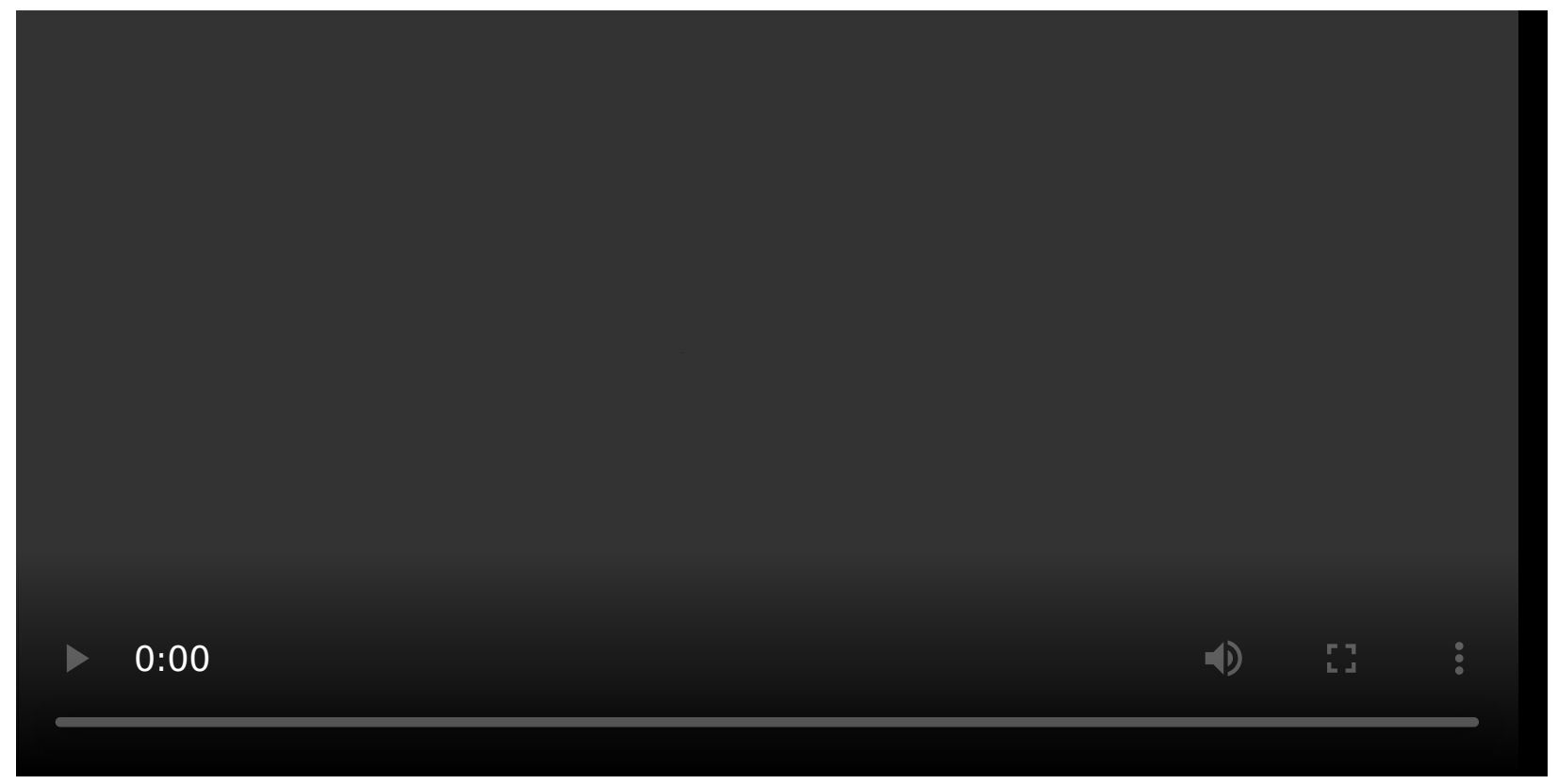




\title{
Aligning practices and spending with issues of diversity, equity, inclusion, and justice
}

\author{
Alexia Hudson-Ward \\ Associate Director of Research and Learning \\ Massachusetts Institute of Technology (MIT) Libraries \\ for \\ Commonplace - a publication of the Knowledge Futures Group
}

\section{Slide 1}

Hello everyone. My name is Alexia Hudson Ward, and I'm the associate director of research and learning for MIT Libraries. This video explores how the library community can strive to align Open Knowledge practices and spending to support diversity, equity, inclusion, and justice. In this video, I will use DEIA as initials to describe diversity, equity, inclusion and justice. The " $\mathrm{A}$ " is for accessibility, an important yet often overlooked aspect of inclusion efforts with open knowledge.

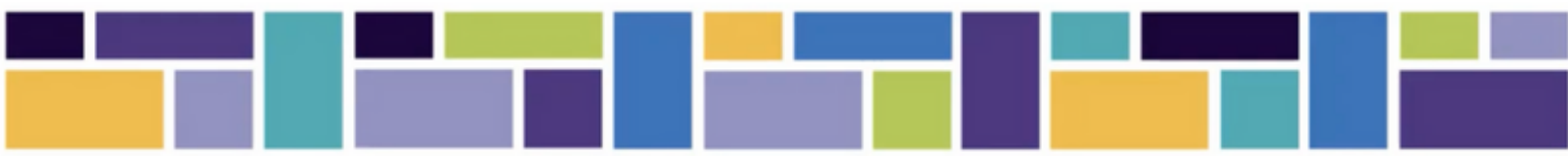

\section{Diversity, Equity, Inclusion \& Accessibility}

\section{Slide 2}

And so everyone, you know, I'll admit that assuring the centrality of accessibility with Open Knowledge practices is a personal blind spot for me, and one that I'm working really hard to remediate. I have this blind spot, despite my professional and personal commitment to inclusive workplace practices within the information professions. So incorporating accessibility into my dialogue points in this video, is a form of 
accountability for me, and for all of us as we explore the important topic of aligning spending to improve inclusive Open Knowledge practices.

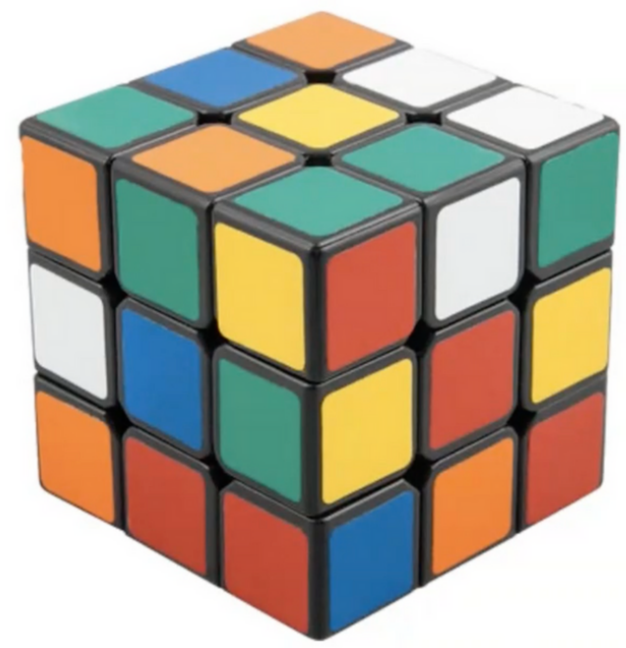

\section{Slide 3}

And everyone, you know, this is a really big puzzle for us to think about, and for us to contextualize. And in the spirit of my institutional location of Cambridge, Massachusetts, in the United States, this matter is what is often referred to in the management literature as a "wicked problem," which has a kind of circular effect, and sometimes individuals believe that it is not solvable. However, I hope that the elements that I share in this video will provide the library community elements to incorporate into an actionable and sustainable framework for us to move beyond the performative, concerning DEIA and open knowledge. 


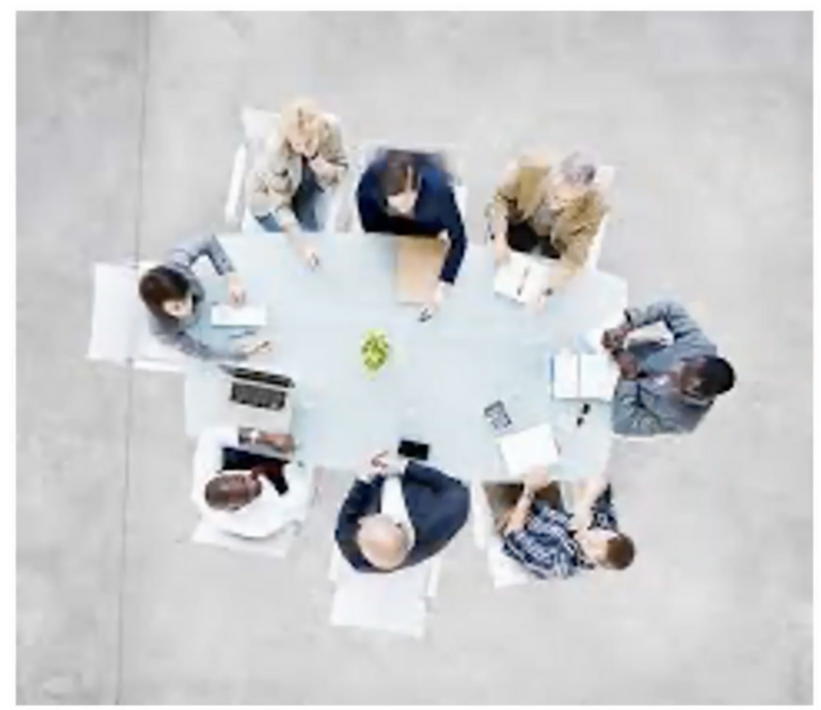

Slide 4

And so, the stated principles of Open Knowledge deeply aligned with the essence of DEIA and justice efforts. Open Knowledge, as we all know, means free, fair, and even possibly hackable solutions to bring forth new dimensions of knowledge creation. This free, fair, non-mediated, and possibly hackable Open Knowledge future is inherently inclusive, and invites all of us to the proverbial table to benefit from, and to contribute to, broadening our somewhat limited views of what constitutes scholarship, scholarly contributions, and knowledge sharing dynamically. And so, how open knowledge practices and DEIA intersect makes me really interested and excited, as I view this opportunity as a natural extension, to the core missions of libraries, of democratizing learning and knowledge. 


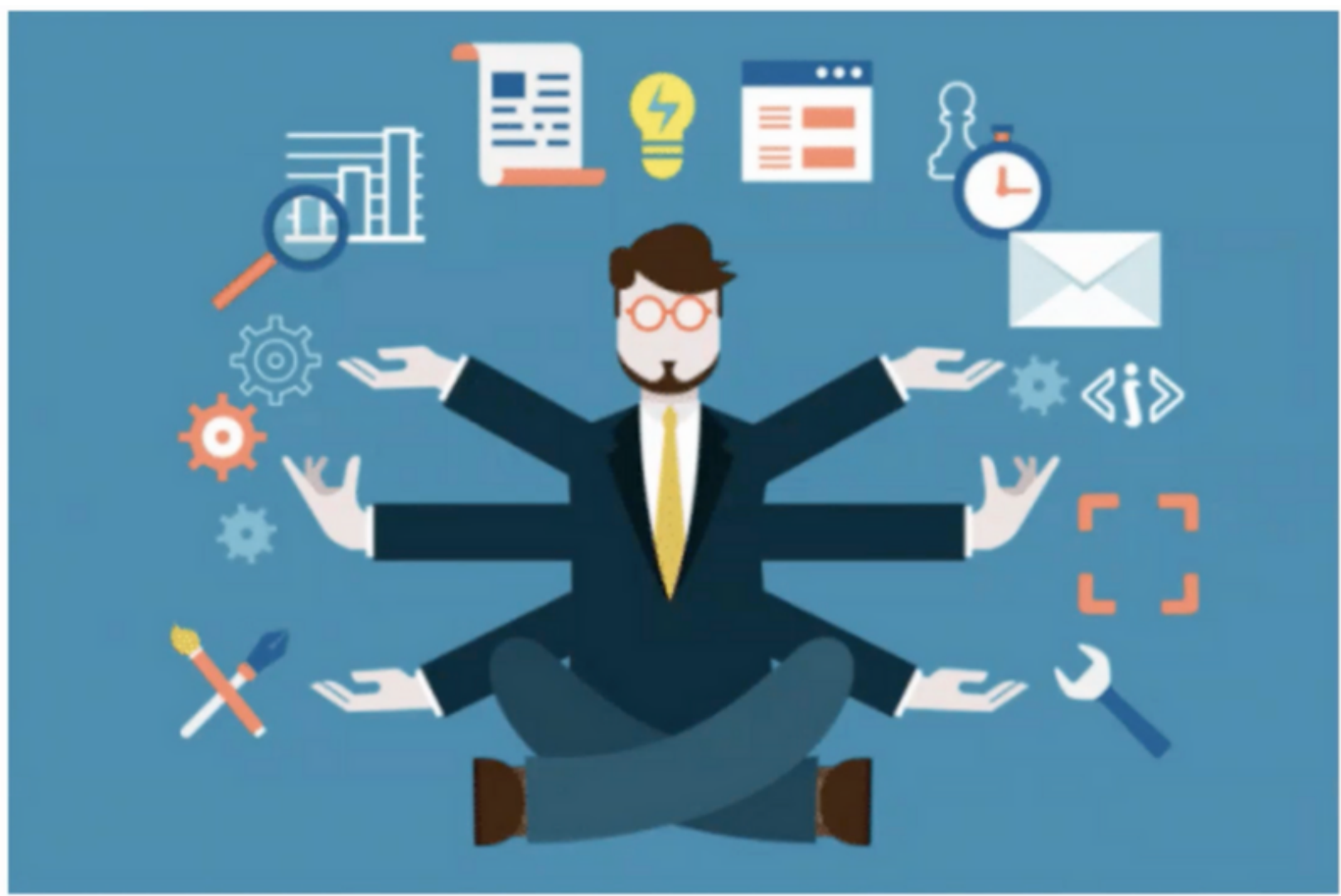

\section{Slide 5}

And it's interesting that yet as a growing global mute movement, that the Open Knowledge community remains relatively closed to diverse scholars and activists. Those that tend to be heralded as leaders within scholarly communication are largely cisgender white people, and oftentimes white males. And far too often, our computational center dialogues regarding Open Knowledge practices privileges such a small subset of scholars and Digital Curation experts that tend to speak the same technical and cultural languages. 


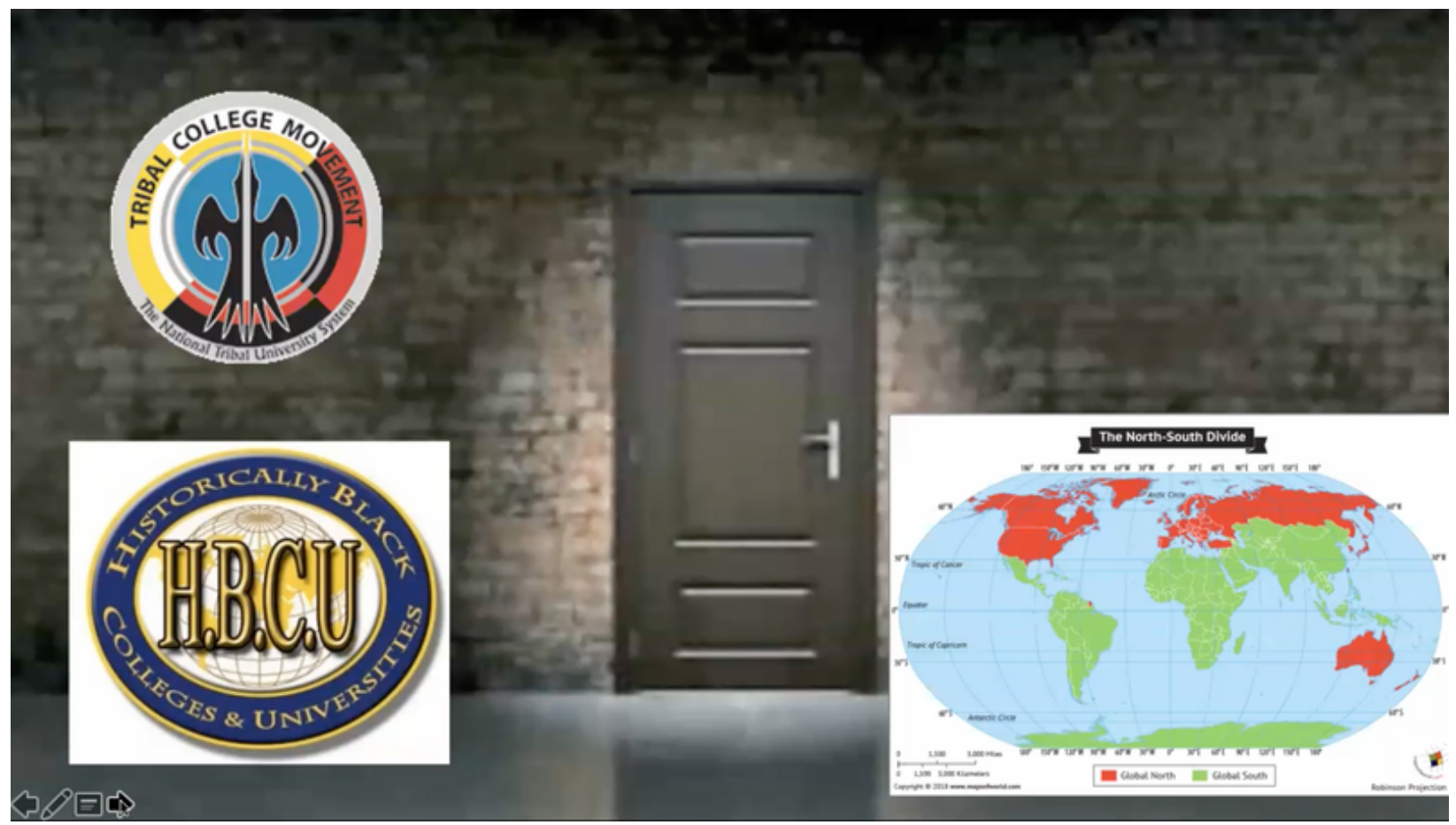

\section{Slide 6}

And so the library community-interestingly-tends to also generally place responsibility on individuals from diverse populations to raise the need for an inclusive Open Knowledge engagement with important entities such as America's historically black colleges and universities, tribal institutions, community colleges, in addition to the region that is frequently referred to as the so-called Global South. And moreover, Open Knowledge accessibility tends to still be treated as like an app or add-on, or an extension, with a significant number of libraries. And so we must ensure that accessibility writ large, that contains solutions that can cross platforms, multiplatform, Open Knowledge Access for a large portion of the world's population, really depends on mobile devices. And so that is their internet on-ramp. So as we think about and contextualize what Open Knowledge practices should be, we have to be more deliberative with incorporating adaptive technologies, and their supporting systems infrastructures, into any plans that we may make. 

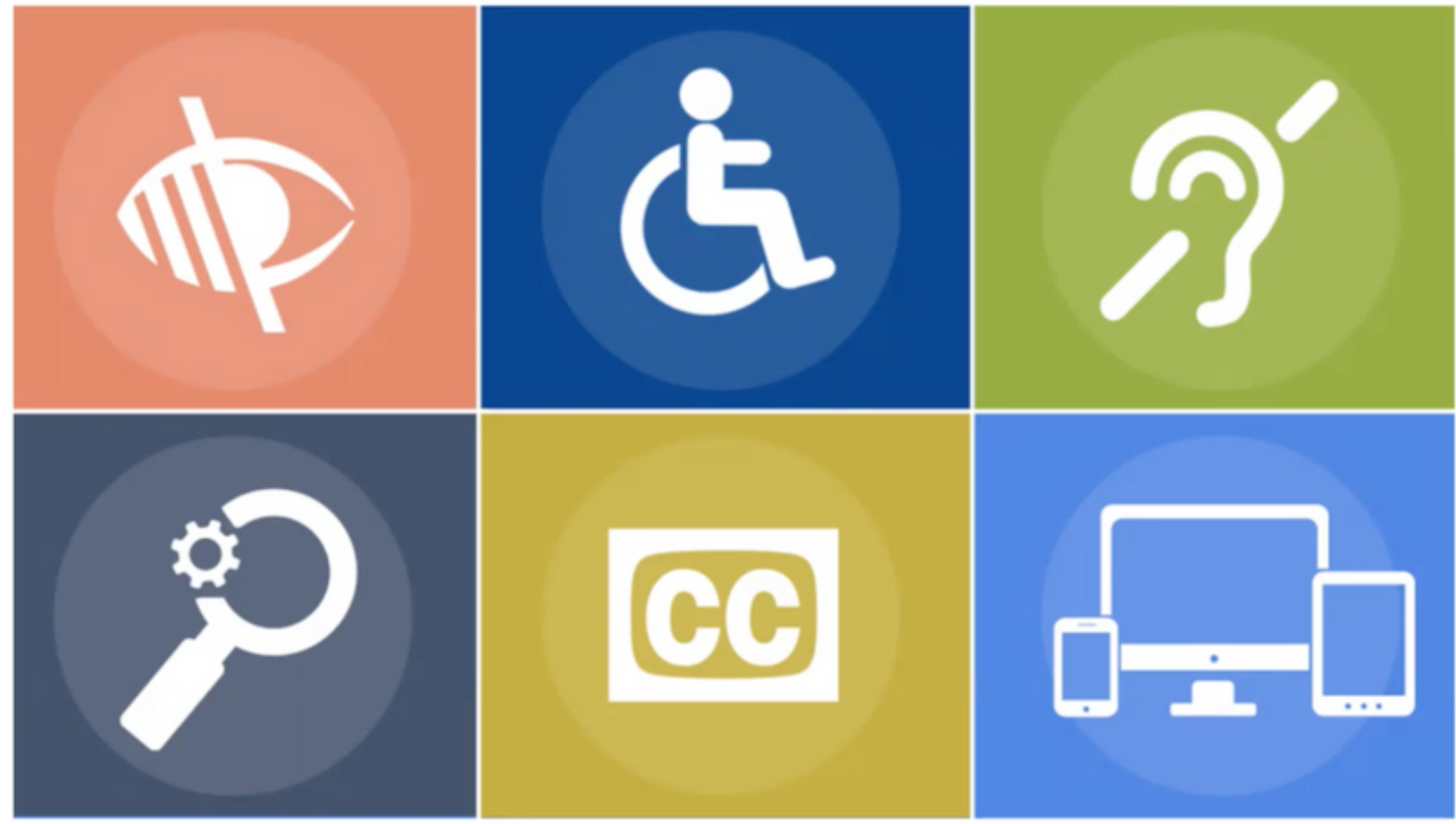

Slide 7

And so, you know, everyone: I've kind of talked a lot about accessibility. And I've talked a lot about, you know, my kind of, you know, high vision, if you will-or ascended vision -in the context of open knowledge. And there are a couple of concepts that I do want to flesh out a little more, that capture some of these elements that I just walked through pretty quickly. And so, if you'll just bear with me, I'm going to go through those now. 


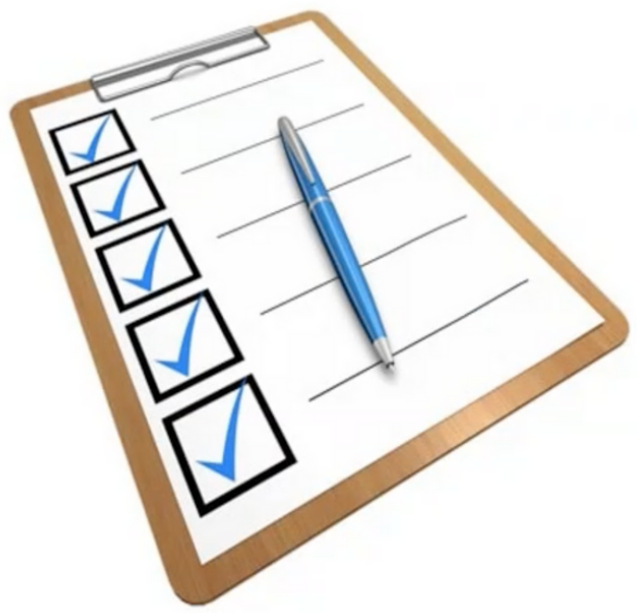

Slide 8

And it's actually almost like a checklist, and you can think about it in that way. You know: here are some of the elements that we need to do within the library's community, to ensure that our spending and our budgetary practices align with this most perfect possible future, of making sure that DEIA and justice activities are centered within our budgetary practices.

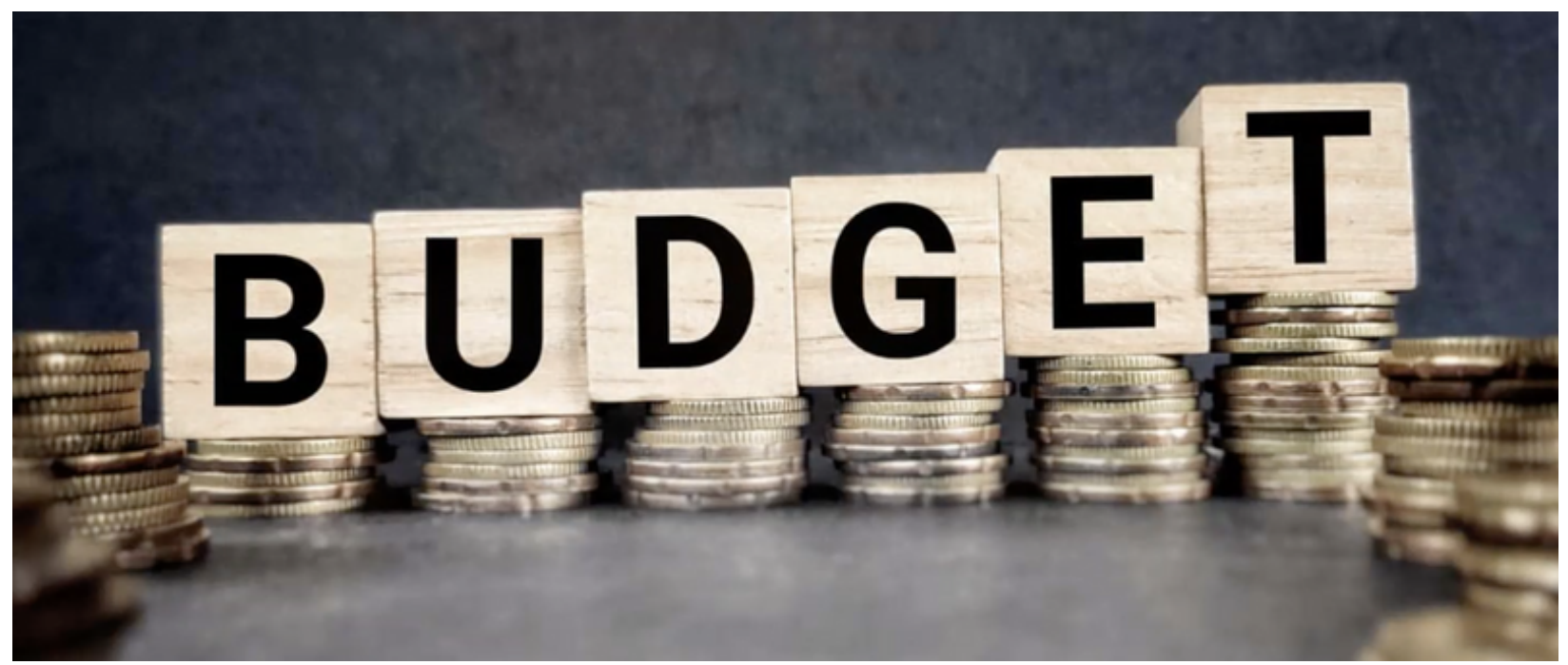

Slide 9

And so, if feasible, everyone, I believe that Scholarly Communications budgets should include somewhere between 5-10\%, of an annual allocation for DEIA centered initiatives. The rationale for this specific percentage, everyone, is for a reason, or for several reasons. And so, I believe it takes about three years, roughly 36 months, for an 
initiative to become embedded practice within any organization. And initiatives really do need that funding ramp, that time, in order to blossom. Libraries can deploy these funds to support a wide range of efforts, such as-you know, as I was just mentioningcreating multi-platform accessible softwares investing in developing and retaining compositional diversity within the scholarly communications pipeline; and also thinking about how you can help to sustain these important special mission institutions within the United States, as an example, the tribal institutions, and also the historically black colleges and universities, often referred to as HBCUs. Because those institutions are still largely responsible for creating new roles and new entry points for people of color, and most specifically within the sciences and within the STEM fields. And so it's important that as we're thinking about diversity initiatives for open knowledge, that we consider some partnerships with those types of organizations.

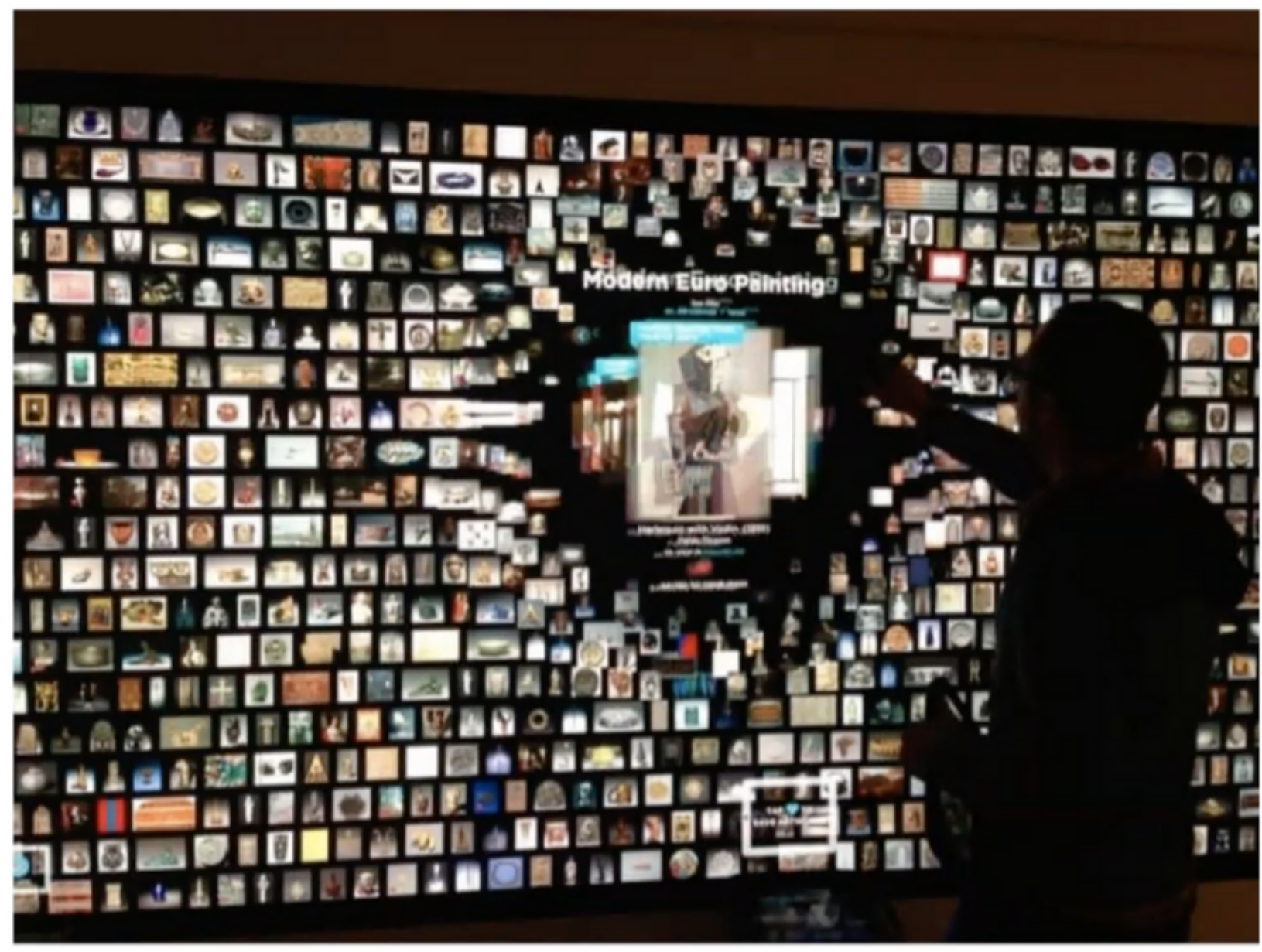

Slide 10

The other thing, everyone, is that, you know, it's important for us to think about partnering with our colleagues in the global cultural heritage community: to 
incorporate objects, images, and various elements of visual culture that is so essential to advancing Open Knowledge, especially in this moment of reckoning, and repatriation, and all of the controversy and work that is happening in relation to objects that have been acquired through illicit means, that are now being returned back to some communities. And yet some other cultural heritage organizations are debating whether or not they should return these images. And so, this is really so important for the library community to have a leadership voice, and also do some work around, in relation to Open Knowledge practices. And so, as many of you know, with the support of some philanthropists and funding entities, you know, some libraries and museums on academic-higher education-campuses, they're creating scaling platforms in support of collection discoverability. These are massive efforts that take a lot of staff, and a lot of dollars.

And those large efforts can and should be supplemented with smaller activities that I think many of us have the potential to scale. And those smaller activities, perhaps, could focus on illuminating a single collection or a single theme. So in the case of maybe an indigenous culture, which a library is very close to, you know, thinking about how, with permission of those communities and in relation to a partnership with a cultural heritage organization, developing a really rich digital platform and a really rich digital series of digital assets to really help to advance objects-based pedagogy in a different way while also leaving the original object to its respective community. And so, there's really just this important piece that I want us to think about, and contextualize in the context of budgeting. You know, how do we make sure that we are doing everything that we can to create a series of digital learning objects, so that we can advance the world's knowledge and partnership with our cultural heritage colleagues, while also honoring the traditions of many of the communities through which some of these objects have come from. 


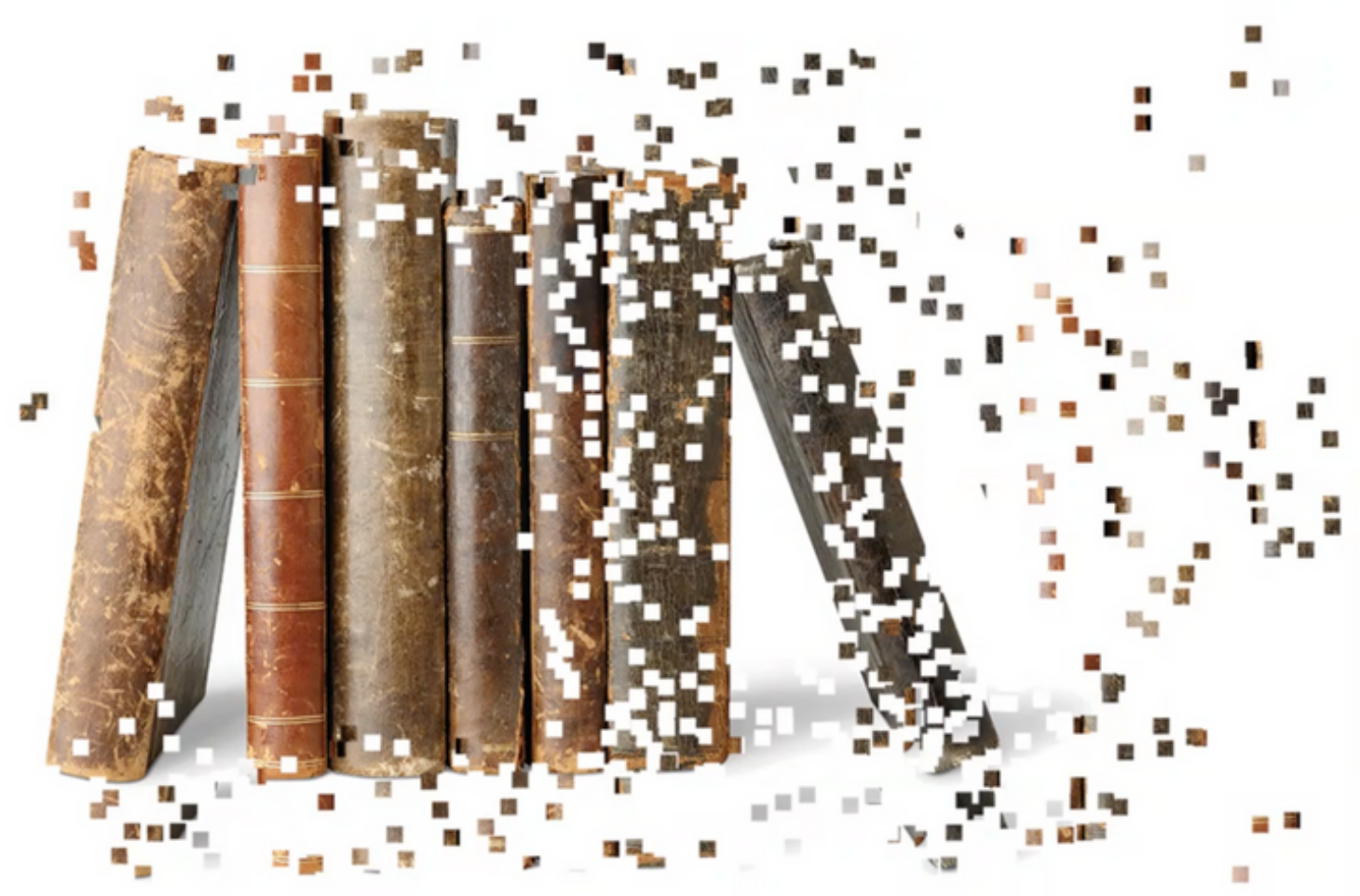

Slide 11

And my last point, everyone, for this video, is that libraries must not abandon financial support of the digital humanities, and what many institutions refer to as "data expeditions" (excuse me for stumbling over that). So, allocating budgetary support for Open Knowledge activities that highlight this kind of dynamic, retrospective gaze of history-like, there's a lot of really interesting and important work happening in relation to slavery cultures, the movement of sacred illuminated texts throughout a multitude of countries-all of that work is really important. I would also add that there is a lot of power in thinking about the ways in which libraries can help to fund, support, and build sustainable platforms, networks, and information around doing some elements about the power of modern communications networks as compelling predictors for a wide range of social phenomena that happens within diverse populations.

So, such as things like tracking voter trends; the impact of health disinformation; or social media: there is really an essential space for libraries to contribute both people power and funding to support data-set production, sustainability, and then ultimately developing a series of repositories and strategies of sharing this detail-these detailsvery broadly with a wide range of communities, both internal and external to higher education. And so, that is something that I really think is important for us to think 
about. We tend to contextualize diversity, equity, inclusion, accessibility and justice in this kind of very linear frame. However, we can think about the ways in which the data is dynamic, the data helps to inform new knowledge; the data can help to enforce or reinforce justice efforts within a wide range of diverse communities. To me, that's how our budgets can most neatly align with DEIA efforts, when we're talking about advancing open knowledge.
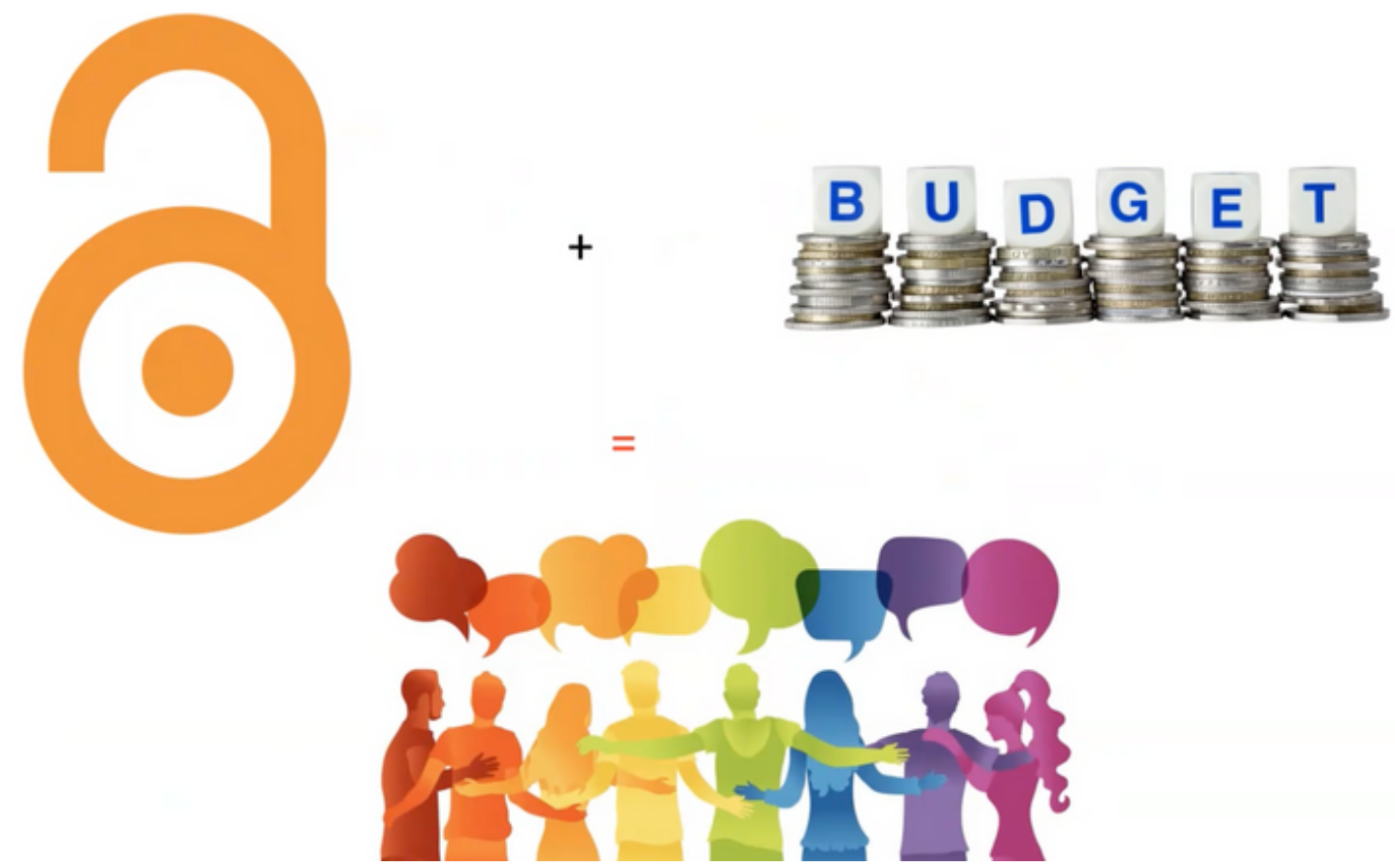

Slide 12

And so everyone, it's really, to me, I tend to think of a lot of these ideas and concepts within, like this idea of an equation, if you will. And so, if we're going to advance Open Knowledge, we have to have the money to do that. Right. And many of us do. And we tend to deploy our dollars in that which we feel is important and essential to the advancement of Open Knowledge in various forms of open access efforts. But I would argue that it is equally important to put within our budget lines some form of DEIA centered efforts. So whether it is a sub line item in your existing budget, or it is a completely different line item in your budget, think again about the point that I raised earlier: $5-10 \%$ over no less than three years. So annually, five to $10 \%$, you know, drawing up 15 to $30 \%$ of your scholarly communications or open efforts budget, to place that towards a wide range of DEIA efforts. And that is how I believe that these efforts will grow, that they will become scalable, that they will become sustainable, and that they will continue to enrich a wide range of scholars and public historians, content 
creators, activists, and other individuals who are dedicated to improving the lives of all of us-and most specifically, communities of diverse populations.

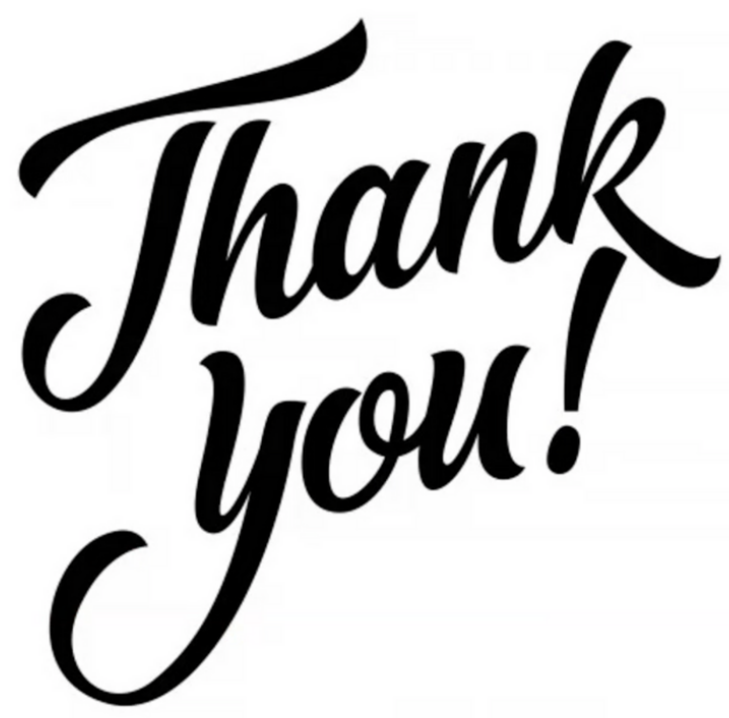

Slide 13

And so, I want to thank you so much for listening to my video. I hope that it has spurred some interest for you and some ideas. Please feel free to reach out to me should you want to dialogue about this more. Of course, I have a lot of ideas and concepts. But I did want to keep this video as refined as possible, to kind of hit the highlights so that it wouldn't be too long or too verbose. So thank you so much for your time. Thank you for engaging in these important conversations. And I look forward to more conversations with you in the future. Thank you. 